\title{
René Maran : genèses de la première édition (1921) de Batouala, véritable roman nègre, et de sa préface
}

\author{
Charles W. Scheel
}

\section{OpenEdition}

\section{Journals}

Édition électronique

URL : https://journals.openedition.org/coma/7748

DOI : $10.4000 /$ coma. 7748

ISSN : 2275-1742

Éditeur

Institut des textes \& manuscrits modernes (ITEM)

\section{Référence électronique}

Charles W. Scheel, « René Maran : genèses de la première édition (1921) de Batouala, véritable roman nègre, et de sa préface », Continents manuscrits [En ligne], 17 | 2021, mis en ligne le 15 octobre 2021, consulté le 18 août 2022. URL : http://journals.openedition.org/coma/7748 ; DOI : https://doi.org/ 10.4000/coma.7748

Ce document a été généré automatiquement le 18 août 2022.

\section{cc) $(1) \odot$}

Creative Commons - Attribution - Pas d'Utilisation Commerciale - Pas de Modification 4.0 International - CC BY-NC-ND 4.0

https://creativecommons.org/licenses/by-nc-nd/4.0/ 


\title{
René Maran : genèses de la première édition (1921) de Batouala, véritable roman nègre, et de sa préface
}

\author{
Charles W. Scheel
}

\section{Introduction}

1 Il s'agit dans cette contribution de faire le point sur la genèse du roman Batouala de René Maran et sur celle de sa préface rédigée par l'auteur lui-même, sur la base des documents disponibles dans diverses archives pour la période entre fin 1909 (départ de Maran pour l'Oubangui-Chari) et juillet 1921 (publication du roman par Albin Michel à Paris). Cette période a été cruciale pour la formation de l'écrivain qui ne s'était signalé précédemment qu'à l'attention d'un public littéraire français restreint, par la parution d'un premier recueil de poèmes en $1909^{1}$, mais qui allait acquérir un statut international de véritable mentor " ès lettres noires », entre son installation à Paris en 1924 et sa mort dans la même ville en 1960.

2 Seront cités les passages les plus significatifs des nombreuses correspondances privées de l'écrivain pendant la douzaine d'années concernées, ainsi que des études faisant référence à des documents réunis dans le dossier de carrière de René Maran dans l'administration des colonies en AEF, conservé aux $\mathrm{ANOM}^{2}$. Ces derniers éclairent plus particulièrement les conséquences de la célèbre préface de Batouala ${ }^{3}$, qui a suscité bien plus de réactions que le roman lui-même, surtout après l'attribution du prix Goncourt en décembre 1921, que certains perçurent comme "scandaleuse ». Mais la présente étude s'arrête en amont de la question complexe - désormais étalée sur un siècle - de la réception de l'œuvre après sa publication en juillet.

Beaucoup de références proviennent du fonds «Hommage de Madame Camille René Maran à la République du Sénégal ", conservé à la Bibliothèque centrale de l'UCAD de Dakar, dont une grande partie est numérisée et accessible en ligne. Et bien des citations de lettres diverses seront familières à qui connaît deux textes fondamentaux dans la 
réception de l'œuvre de René Maran : la longue préface de Léon Bocquet pour le conte Le Petit Roi de Chimérie (1924) et "La genèse de Batouala ", l'étude de Manoel Gahisto, dédicataire du roman, parue dans l'ouvrage Hommage à René Maran (1965, Présence Africaine). Mais ces deux ouvrages ne sont conservés que dans de très rares bibliothèques ${ }^{4}$.

4 Par contre, de nouvelles sources archivales - notamment celles des Bibliothèques de la ville de Bordeaux et celles des ayants droit de René Maran ${ }^{5}$ - sont en cours de numérisation pour un site René Maran mis en ligne sur la page MANIOC des bibliothèques de l'Université des Antilles et de l'Université de Guyane. L'étude génétique détaillée du roman est, elle, en cours pour une autre publication ${ }^{6}$. Elle pourra s'appuyer sur deux avant-textes connus à cette heure : un manuscrit conservé dans les archives des Bibliothèques municipales de Bordeaux (BBX19104), et un tapuscrit conservé dans le fonds d'archives familiales René Maran. La présente étude se concentre sur les conditions de la rédaction du roman Batouala dans la période 1913-1919, et sur les conditions de son édition et de l'insertion de la préface, entre 1919 et la parution du livre en juillet 1921 avec le sous-titre « Véritable roman nègre » chez Albin Michel.

Fig. 1 : Feuillet tapuscrit, incipit de Batouala le Mokoundji

\section{RM_BM_BATOUALA_TPS1}

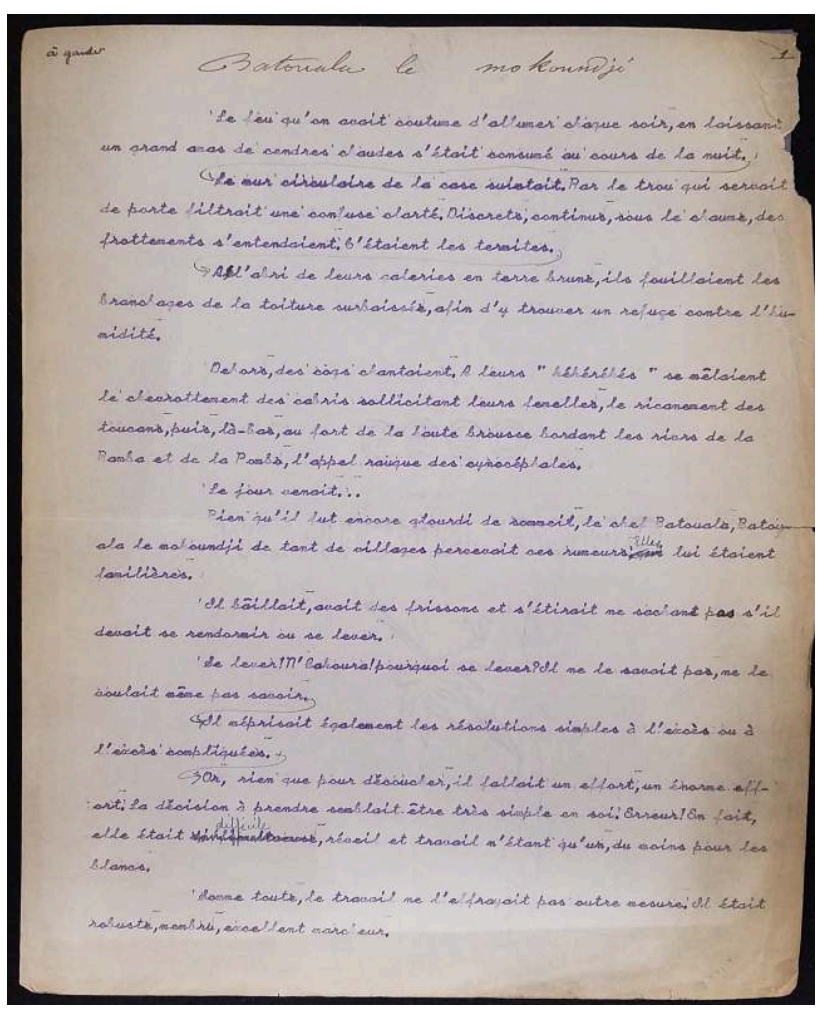

Archives familiales René Maran

Partir en 1909, à l'âge de 22 ans pour rejoindre son père dans le service colonial en Afrique a manifestement constitué une grande aventure pour René Maran ${ }^{7}$ qui laissait sa mère, deux jeunes frères et beaucoup d'amis à Bordeaux où il vivait depuis 15 ans, sans escapade connue en dehors de compétitions sportives. Cette séparation brutale a rapidement suscité une activité intense d'épistolier et les innombrables lettres du jeune 
homme offrent une mine d'information sur ses activités et ses états d'âme pendant les voyages et séjours qui allaient rythmer sa vie pendant plus de douze ans jusqu'à son retour définitif en France en 1923. Parmi les correspondants figurent André Lambinet, le professeur de lettres de René Maran au Lycée de Bordeaux, qui demeurera l'interlocuteur privilégié pour tout ce qui concerne l'expression poétique, ainsi que Manoel Gahisto et Léon Bocquet (ce dernier, éditeur du premier recueil de poèmes de Maran en 1909), deux hommes que Maran n'avait jamais rencontrés avant son départ, mais qui deviendront de grands amis.

6 Mais il y a aussi ses amis de la "Confrérie de Sainte Poésie », comme se désignait le groupe de jeunes poètes bordelais autour d'André Lafon et d'Auguste Pujolle, dont certains étaient aussi, comme Maran, des amateurs de rugby, de football ou d'escrime. Le ton des lettres est amical, complice et libre - parfois même " déboutonné », comme dans celles adressées au jeune Charles Barailley, connu un soir chez Lambinet pendant le bref séjour de Maran à Bordeaux en 1911. Mais au-delà du style, ce qui ressort de manière frappante des extraits de ces correspondances, cités par Léon Bocquet dès sa préface de 1924 et bien plus tard par Manoel Gahisto, c'est que «l'Afrique a été le creuset de plusieurs œuvres $»^{8}$ de genres divers (poèmes, romans, contes, essais, études littéraires), esquissées en parallèle pratiquement dès l'arrivée de Maran à l'escale de Brazzaville à la fin de 1909.

7 En d'autres termes, loin d'être isolée dans un lieu et un temps spécifiques restreints, la genèse de Batouala s'est opérée sur six longues années au sein de genèses multiples. Si le poète en herbe s'était manifesté dès avant le départ pour l'Afrique, le talent littéraire multiforme qui s'affiche clairement dans la liste de la trentaine d'œuvres publiées par l'écrivain en cinquante ans de carrière, s'est épanoui pendant son séjour en A.E.F., avant même la publication du premier roman qui allait le consacrer - avec bruit et fureur.

\section{La genèse selon Léon Bocquet}

Dans la préface du Petit Roi de Chimérie, Léon Bocquet fait une synthèse des thèmes qui reviennent dans les correspondances de Maran. Il donne aussi des éléments de biographie pour réhabiliter Maran dont on sait que les qualités d'écrivain avaient été remises en cause par certains qui prétendirent qu'il était "scandaleux» que le Goncourt fût remis à un auteur inconnu, noir de surcroît, qui écrivait mal et osait critiquer la colonisation française alors qu'il faisait partie de son administration. Bocquet rappelle non seulement les prix de grammaire de l'élève Maran mais aussi ce que la grande majorité des amateurs de romans ou lecteurs de journaux en France ignoraient : avant même de partir pour l'Afrique fin 1909, un recueil de poèmes de Maran avait eu l'honneur de paraitre dans la "Bibliothèque du Beffroi ", chez un éditeur exigeant (lui, Bocquet...) qui venait de quitter Lille pour s'installer à Paris (p. 7-9).

9 Sont alors citées les premières lettres que Maran lui adresse, alors qu'il remonte le fleuve Congo depuis Brazzaville vers son poste à Bangui : « Je vais bien loin. Je suis un peu fier, un peu triste aussi ", écrit Maran le $1^{\text {er }}$ février 1910, avant d'ajouter ces lignes significatives de son malaise dans le contexte colonial : «Car maintenant, avec un cœur français, je sens que je suis sur le sol de mes ancêtres, ancêtres que je réprouve parce que je n'ai pas leur mentalité primitive ni leurs goûts, mais ce n'en sont pas moins des 
ancêtres ». Selon Bocquet, « Dès cet instant, la dualité qui s'affirme dans Batouala et qui va partager René Maran [...] va s'accuser en lui et lui imposer dans toute sa pressante acuité le problème et le conflit des races » (p. 15).

Après une mise en perspective de certaines accusations portées contre Maran, qui aurait fait preuve d'une "sévérité particulière vis-à-vis des indigènes " dans ses fonctions de commissaire de police à Bangui, Bocquet mentionne un fait important pour la carrière de Maran: la mort de son père, qui venait à peine de prendre sa retraite à Bordeaux, et qui motive un pénible et bref retour en France fin 1911. Lorsque Maran revient en mai-juin 1912 à son poste de Grimari, où il avait été muté en juin 1911, à sa demande, il raconte, dans une lettre à Bocquet en août 1912, une tournée en brousse dans cette "région la plus saine de l'Oubangui et la plus riche", et mentionne des conflits de l'administration avec certaines «populations hostiles mal pacifiées et guerrières " ainsi que les dangers que constituent les bêtes sauvages (p. 17-19). Maran avait découvert le cadre de son roman Batouala et des œuvres subséquentes de ce que j'appelle son «Cycle de la Brousse africaine».

Des détails sont fournis dans les lettres suivantes à Bocquet : des anecdotes sur la vie sauvage (comme un chien de Maran, emporté une nuit par une panthère, entrée dans sa case alors que lui dormait), la description du personnel de Maran à Grimari, son cuisinier et ses «boys» (p. 20-23). Bocquet cite aussi une lettre de Maran à Gahisto de juillet 1913, qui inclut de très beaux passages descriptifs de la nature, et dans laquelle est mentionné pour la première fois le titre du manuscrit de roman sur lequel Maran travaille déjà : « Il faudra que je note ce soir les effets de lune dans l'orage, Batouala-leMakondji ${ }^{9}$ bénéficiera de ces observations » (p. 27).

Bocquet aborde ensuite la période difficile de l'entrée en guerre, qui déchire Maran car il souhaite s'engager dans l'armée mais n'en obtient pas l'autorisation. Puis il est secoué par la nouvelle de la mort de sa mère à Bordeaux, qui lui parvient le $1^{\mathrm{er}}$ janvier 1915. Maran écrit qu'il a «le spleen », qu'il « se brouille avec ses livres ». Après le congé qui lui est accordé d'août 1915 à mars 1916 pour s'occuper de la succession à Bordeaux (il est désormais seul responsable de ses deux jeunes frères), il s'octroie quinze jours à Paris où il rencontre, enfin, Léon Bocquet et Manoel Gahisto.

De retour en Oubangui, quand le travail le lui permet, il se replie sur sa bibliothèque itinérante (une centaine de volumes) et les nombreuses revues auxquelles il est abonné. Il est très bien informé et Bocquet estime que « Ses lettres offrent un intérêt littéraire considérable » (p. 37). En effet, Maran y discute les thèmes et les aspects de langue et de style de toute une série d'auteurs, des classiques jusqu'à Bergson et Tagore.

Bocquet évoque ensuite le travail intense et patient de Maran sur Batouala, et cite une lettre du 4 juin 1917 : «[...] mon récit apportera une note nouvelle dans la littérature. C'est de l'exotisme vécu, vrai, cru, [...] en excellent français. Je n'ai même pas cru qu'il fût nécessaire d'user du petit nègre»(p.41). Bocquet ne commente pas cette affirmation de Maran qu'il faut pourtant nuancer : dans Batouala - et dans les autres romans et contes du cycle africain - il y a du " petit nègre » chaque fois que Maran fait parler les kapitas qui n'ont pas appris le français à l'école et parlent un sabir oral, certes, mais essentiel dans la communication quotidienne entre colonialistes blancs (ou noirs, comme Maran) et indigènes ${ }^{10}$.

15 Les pages 43-49 sont consacrées par Bocquet à des réflexions pénétrantes où il distingue entre la préface de Batouala, qualifiée de "préambule fâcheux, certes ", et le roman, deux documents qui ne sont pas contemporains. Pour Bocquet, le roman est un 
"document ethnique ", dans lequel "Maran s'est penché sur les âmes obscures qui l'entouraient », et qui relève de la retranscription, de l'observation et de la traduction de la langue banda parlée par les protagonistes ${ }^{11}$. C'est un "procès-verbal littéraire ", car le texte est basé sur les longues conversations avec le « vrai» Mokoundji (un certain Goara) : "René Maran dessine d'après nature ", si bien que Bocquet estime ridicule l'accusation de plagiat d'autres romans coloniaux qui allait être portée contre Maran après le «scandale du Goncourt $»^{12}$.

16 À propos de la Préface de Batouala, Bocquet estime qu'elle est l'expression de l'aigreur et de la colère de Maran, développés depuis plusieurs années dans ses relations souvent tendues $^{13}$ avec l'administration coloniale, au sein de laquelle il a pu cependant constater des " comportements blancs » différents au fil des ans. Pour Bocquet, «Les deux documents d'apparence illogiques, le roman et son préambule, ne relèvent pas de la même inspiration » et appartiennent à " deux temps différents de la question nègre et coloniale, vus à travers un tempérament impressionnable à l'excès » (p. 56-57). Dans sa conclusion de cette longue section, et avant d'en arriver à sa courte préface proprement dite au Petit Roi de Chimérie, Bocquet ajoute que Maran «n'avait jamais osé espérer semblable retentissement auprès de son livre [Batouala] et auprès de son nom " (p.57). On verra, dans d'autres lettres de Maran, qu'une telle opinion peut paraître lénifiante : le romancier savait qu'en insérant sa préface, Batouala deviendrait un brûlot - voire le souhaitait.

\section{La genèse selon Manoel Gahisto}

Autant le témoignage de Léon Bocquet était précoce (car l'œuvre publiée du romancier ou conteur Maran n'en était qu'à ses débuts, en 1924), autant celui de Manoel Gahisto, dédicataire du roman dès 1921, était tardif - mais bien plus substantiel, documenté et précis. En effet, sa «Genèse de Batouala » ne parut qu'en 1965 chez Présence Africaine dans le volume Hommage à René Maran - c'est-à-dire cinq ans après la mort de Maran et dix-sept après celle de Gahisto lui-même ${ }^{14}$.

Avec plus de cinquante pages, cette remarquable étude constituait l'hommage le plus substantiel. En guise d'ouverture, Gahisto compare le début du roman dans la version de 1921 avec celle, dite définitive, de 1938, et constate beaucoup de remaniements minutieux, faits "en Europe, parmi les livres», car Maran est "un homme que tourmente la passion de la langue ». Partant de là, il souhaite « reconstituer, d'après la correspondance que le jeune écrivain entretenait avec ses amis, la genèse de Batouala, dans l'atmosphère africaine du moment » (p. 94).

Parmi les correspondants de Maran, le premier récipiendaire d'une lettre (datée Brazzaville, le 13 janvier 1910), cité par Gahisto, est Paul Culine, un proche voisin des Maran à Bordeaux, boucher de profession mais également amateur d'escrime, de rugby et de poésie, "auditeur attentif, qui gardait précieusement copie de certains morceaux » que lui confiait le poète débutant Maran. Le ton enjoué et chaleureux des messages que ce dernier lui envoyait en dit long sur la qualité de l'intégration du jeune Maran dans le tissu social bordelais, et l'un des premiers textes rédigés par Maran à Bangui sera un long poème («le plus noble et le plus douloureux qu'ait jamais conçu mon cerveau ») dédié à « l'un de leurs commensaux de jadis [à Bordeaux], Grive, pauvre garçon maladif » dont Culine lui apprit le décès (p. 97). 
ahisto souligne que les lettres de Maran, dès ses premiers mois en Afrique, montrent à quel point le travail littéraire se développe - parallèlement à celui du fonctionnaire colonial - sur plusieurs fronts à la fois, et dans un échange intellectuel et/ou affectif constant avec les correspondants en France - sachant qu'il fallait attendre au moins quatre mois pour obtenir une réponse à tout courrier. Le fait le plus notable qui fascine Gahisto est le basculement presque immédiat de Maran vers la prose pour évoquer le cadre africain, alors que la poésie reste le média de l'expérience intime et du souvenir. Ainsi cite-t-il une lettre dans laquelle Maran annonce de "prochains livres», dont un recueil intitulé - de façon très parlante - «Le Chemin de la solitude », et ajoutant : «Je vous donnerai aussi mon roman africain "Samba Diallo". Il est un peu monotone, à cause du sujet développé. Mais il est écrit dans un français limpide, substantiel, classique... » (p. 98) ${ }^{15}$. Et Gahisto de s'interroger sur la capacité de Maran de passer aussi rapidement de l'art du vers à celui de la prose - avec raison, puisque ce premier projet de roman a manifestement été bientôt abandonné et qu'aucun manuscrit n'en a été retrouvé.

21 Après avoir cité une lettre à Culine de novembre 1910, dans laquelle Maran exprime sa douleur suite à l'annonce brutale du décès de son père ${ }^{16}$, Gahisto mentionne une lettre à lui adressée depuis Diouma le 19 juin 1911, dans laquelle un «René Maran joyeux décrit la brousse réelle » avec des vers et des croquis de cases, car il a réussi à se faire muter dans la brousse après « 17 mois à Bangui, l'insalubre ». 1911-1912, mais si souffrant et empêtré dans les soucis financiers qu'il ne peut ni se rendre à Paris ni faire imprimer "Le Songe d'une nuit d'automne", son nouveau recueil de poèmes. Plus directement intéressants pour le thème de la présente étude sont les passages suivants: «Je consulte mes notes prises en Afrique, je les mets au propre. [...] L'exotisme du Roman d'un métis n'est pas de l'exotisme pour salons [...] Doisje le proposer à Paris-Journal ? » (10.01.1911) et «J'ai mis le point final hier à mon roman exotique. Vous avez raison. Je l'appellerai Djogoni sans plus. [...] Je vais commencer le roman autobiographique qui ira de mes 6 à mes 20 ans » (18.01.1911). Gahisto ajoute que Maran est reparti pour l'Afrique sans avoir pu attendre la sortie de La Vie intérieure et sans avoir envoyé de manuscrit de roman à un éditeur. Mais ce sont dorénavant deux œuvres en prose sur lesquelles travaille le poète publié: l'une sur sa jeunesse bordelaise, l'autre sur son expérience vécue présentement en Afrique.

À partir de cette période, Gahisto est manifestement devenu un correspondant-clé de Maran pour ce qui relève de la prose, et les lettres des trois années suivantes - un séjour ininterrompu en Afrique entre 1912 et 1915 pour Maran - mêlent constamment les considérations existentielles, les notes d'atmosphère et les questions littéraires. Ainsi Maran écrit-il depuis Grimari : «... plus de «veillées studieuses sous la lampe : j'écoute les rumeurs, les tams-tams. [...] Je retouche sans hâte, Djogoni, et j'ébauche un petit acte en vers et une suite de poèmes : Les Belles Images ${ }^{17}$. [...] Orages, siestes, belles nuits de lune... » (01.08.1912). Il écrit aussi aux autres membres de la "Confrérie de Sainte Poésie", devenus contributeurs comme lui de la revue Burdigala: Auguste Pujolle, les frères Lafon, Auguste Hourcade, etc.

Puis Gahisto cite trois grandes pages d'une très belle lettre sur la brousse et la vie coloniale de l'administrateur Maran qui dit songer à «entrer dans une entreprise commerciale pour aider les miens plus efficacement » et qui doit partir en tournée avec vingt miliciens «contre un Langouassi nommé Zagba, chef réfractaire que je veux 
mettre à la raison... » (11.09.1912). Gahisto ajoute à cela une page de commentaires pénétrants sur la différence d'inspiration entre « Djogoni, une histoire de faubourg [de Brazzaville]» et «la matière de nature abondante dont Maran dispose [dans la brousse] ", et qui va orienter son imaginaire vers un autre projet de roman (p. 115).

En effet, dans une lettre du 13 novembre 1912 (le même jour que celui de la rédaction de la lettre à Léon Bocquet, déjà citée) depuis le village de Mabingui, après quelques mois à peine "en brousse ", Maran signale qu' "[il a] entrepris deux proses qui [l]e charment également : l'une sur Mathurin Régnier [poète de la Renaissance] ; l'autre est un roman qui sera purement indigène ", et pour lequel il a déjà un concept et un titre en tête: Batouala, le mokondji (p. 116). Et dès 1913, il envoie régulièrement des extraits tapuscrits pour montrer à Gahisto et à Bocquet, ses mentors en prose, son avancée dans la rédaction de Batouala, bien avant l'envoi final à Henri de Régnier en 1920.

Gahisto mentionne que Bocquet avait reçu, en effet, le premier chapitre de Batouala au début de 1913 et que Maran lui promettait le deuxième dès fin avril. Gahisto cite aussi une lettre que Maran lui avait adressé, à lui, le 8 avril depuis le centre sanitaire de Bandoro (Fort Crampel) où il était allé se faire soigner « après $260 \mathrm{kms}$ dans une région peuplée de fauves et de pachydermes ", et dans laquelle il lui confiait que : «moi, qui ne prise que fort peu les émotions cynégétiques, ces randonnées m'effraient toujours » (p. 118). Gahisto consacre alors plusieurs pages à l'évocation d'autres romans coloniaux et du thème du "poison des tropiques dans la moralité des héros", ajoutant que «Batouala en laissera percer quelque chose par moments mais Maran, lui, lutte»; et que si Maran ne mentionnait pas toujours le roman dans ses lettres, il y était « prodigue de pages évocatrices, dont il ne restera rien [dans le roman], et que, manifestement, « il se faisait la main » (p. 121-123).

Les lettres des mois suivants évoquent surtout des problèmes de santé, l'abrutissement dû à la quinine, le sentiment d'ennui, «d'anémie cérébrale ", et le regret d'un « amour partagé, à la fois intellectuel et sensuel » comme il en avait connu un à dix-huit ans, car il ne pourra «jamais comprendre ni jamais aimer la femme indigène, inerte et simple réceptacle de spasmes désenchantés. Hélas !». Ce thème de l'amie rêvée revient dans une lettre à Bocquet du 26 novembre 1913, depuis Fort Sibut, où il a obtenu d'être muté et va mieux :

Ce mois-ci, j'ai été en proie au démon. J'ai aligné et corrigé des stances qui participeront à mon Livre du souvenir ; j'ai revu et mis à nouveau sur l'établi, des chapitres de Batouala le moukondji et de Une Amie. Bref, je n'ai pas perdu mon temps. (p. 125)

Gahisto cite d'autres lettres à Bocquet de mars et juin 1914, d'un Maran de retour à Grimari et travaillant sur plusieurs œuvres : « Djogoni, un roman colonial (j'ai mis eauxfortes en sous-titre) est complètement fini. Je l'avais commencé en 1910... Aux heures de souvenance mélancolique, j'affine les chapitres et les ébauches du petit roman psychologique Une Amie dont le héros, à certains moments, me ressemble à s'y méprendre " (et qui est sans doute une première ébauche de ce qui deviendra Un homme pareil aux autres), ainsi que divers poèmes.

Gahisto cite ensuite de longs passages d'une lettre très riche, datée du 13 avril 1914, que Maran lui envoie du poste de Griko et qui comportent deux pages de plaintes sur sa fatigue et son exaspération avec tout : les déplacements incessants en brousse (comme à Kouki, où, « heureusement que les indigènes sont peu commodes. Je me distrairai en essayant de les subjuguer... ») ou les exigences financières de ses jeunes frères, alors 
que lui se prive de tout, "n'a plus de flanelles ni de caleçons» et vit "comme un gueux ». Bref, ses travaux n'avancent plus et il déchire tous les articles qu'il commence pour Burdigala, alors pourtant que sa "santé est excellente» et que l'un des trois grands quotidiens de Bordeaux « a consacré une longue colonne à la louange de La Vie intérieure » qui venait de paraître.

Dans un autre passage Maran revient sur Djogoni, ce roman qui est fini, «bien fini », mais dont il se demande s'il le livrera jamais à l'impression.

Ce n'est pas que je sois mécontent de sa tenue littéraire, de son extrême condensation. [...] Je me reproche d'y avoir peut-être mis trop d'imagination, trop d'anticipation. [...] J'exagère. Je n'ai fait que grossir, idéaliser des constatations que tout le monde peut faire - et que j'ai faites. J'hésite toujours... / M. Marius-Ary Leblond m'a - ou m'ont - écrit une très charmante lettre. Ils m'ont dit qu'ils s'emploieraient ferme à soutenir mon effort. Ils m'ont engagé à persévérer. Ils ignorent que je suis totalement vidé. / Un mois de France, avec les notes que j'ai, et Batouala le Mokondji serait terminé. » (p. 129-131).

Maran ajoute toute une page d'observations sur les coutumes des noirs (danses du coït, excision, circoncision, culte du phallus, pratiques funéraires...), dont Gahisto estime qu'elles auraient leur place dans une ethnographie de l'Oubangui-Chari-Tchad. Il ajoute avoir rappelé dans sa réponse à Maran que « le genre roman exigeait de l'action, une intrigue... ", ainsi qu'avoir formulé quelques considérations sur le métissage, " question qui bloque Maran dans Djogoni ...» (p. 232-133) ${ }^{18}$.

Les correspondances de la seconde moitié de l'année 1914 sont évidemment très perturbées par le début de la Première Guerre mondiale, et Gahisto cite une lettre de Maran, envoyée le 18 décembre depuis le poste de Mobaye sur l'Oubangui, qu'il ne reçut que le 11 février 1915, dans laquelle il n'est guère question de littérature, mais plutôt d'opérations militaires. Maran, qui avait été très malade, semble approuver la décision du ministère des Colonies, d'interdire aux fonctionnaires, soucieux comme lui, de rentrer en France :

Quoique, à mon point de vue, je regrette cette décision, je reconnais qu'on a sagement agi. L'abandon momentané de la colonie aurait produit les plus fâcheux effets sur les indigènes. [...] Ces peuplades, qui sont toutes encore foncièrement anthropophages, oublient vite. [...] Et cela eût été désespérant de recommencer ce qui avait été fait, et bien fait. (p. 133-134)

Après la maladie et les affres de l'entrée en guerre, vécus à distance, c'est le décès de sa mère qui allait détourner Maran du travail littéraire, en lui imposant, dans un contexte difficile, la préparation d'un voyage de retour, lequel devait néanmoins, une fois réglées les affaires familiales à Bordeaux, lui permettre enfin de rencontrer Bocquet et Gahisto, venus l'accueillir à la gare d'Orsay le 11 septembre 1915. Gahisto mentionne leurs échanges animés durant les dix-sept jours du séjour de Maran au quartier Latin à Paris, "mettant au point des questions abordées au caprice des échanges de lettres, poursuivis depuis cinq ans " (p. 135) ${ }^{19}$. Gahisto cite ensuite des lettres envoyées depuis Bordeaux dans lesquelles Maran fait part de détails sur son travail concernant Djogoni et Batouala (malgré les accès de fièvre, lorsqu'il néglige de prendre sa quinine), et souligne que Maran refuse de publier des extraits de ces œuvres, qu'il n'estime pas encore au point.

De retour en Oubangui-Chari dès avril 1916 (mais affecté à Fort Sibut et sans se douter qu'il « en aura » à nouveau pour trois ans et demi), Maran mentionne dans une lettre du 14 août à la fois des détails concernant sa vie intime ( ... mes rapports avec les 
femmes d'ici [...], un contact d'épiderme, rien de plus... ») et des commentaires sur ses travaux littéraires :

Je vous envoie le troisième chapitre de Batouala. Dites-moi si vous sentez et croyez voir ce que j'écris. [...] Ma plus grande joie serait que tous ceux qui m'auront lu croiront connaitre aussi bien que moi les coutumes dont je parle, et les pays que j'ai vus, tels qu'ils sont malgré mon désenchantement. (p. 139-140) de Maran "avec trente ans de recul», qu'en ce qui concerne Batouala, Maran fait preuve "d'une persévérance dont les médiocres sont incapables", ajoutant que, «en fait, les versions des chapitres reçus au fur et à mesure, diffèrent très peu » du texte publié en 1921. Il cite ensuite une lettre de juin 1918 dans laquelle Maran dit « manquer de courage pour recopier la fin du roman, qui est achevé », car il pense - déjà - " aux personnages de contes et de légendes" qu'il mettra en action dans Le Petit Roi de Chimérie, « où toute l'Afrique Équatoriale est oubliée » (p. 149-150). cette guerre fait du bien à mon roman colonial. [...] Les Blancs écopent. Encore une fois, je suis totalement absent de ce que je relate. [...] Je ne fais que traduire en français des conversations surprises ou entendues. [...] Je vous envoie le chapitre $4 »$ (p.140). En janvier 1917, toujours depuis Fort Sibut, Maran annonce qu'il pense " pouvoir envoyer, à peu près au point, les ch. 5, 6 et 7 ", mais Gahisto mentionne qu'ayant connu des frictions avec la hiérarchie à Fort Crampel, Maran favorise les déplacements dans le Tchad - ce qui implique une moindre disponibilité pour l'écriture. qu'on ne soignera jamais assez », mais aussi : « c'est la solitude qui m'anémie et me tue. Elle seule» (p. 143). Le 11 mai, il écrit que « les 3 derniers chapitres de Batouala vont bien », mais le 5 juin il annonce que « les derniers chapitres de Batouala ayant cessé de me paraître passables, je les ai, une fois de plus, déchirés » et ajoute ce commentaire déprimé : «Je ne ferai jamais un bon romancier ni un bon conteur. Je suis encore plus difficile pour ma prose que pour mes vers. C'est lamentable» (p. 144), avant de rédiger deux belles pages sur sa vie à Fort Crampel et les attaques de panthères...

D'autres lettres de juillet et octobre 1917, citées par Gahisto, montrent que Maran persiste cependant à travailler sur Batouala (tout en mentionnant un drame en trois actes, Les Timides, qu'il dira avoir détruit peu de temps après, et un autre projet de roman "à la fois imaginé et autobiographique", intitulé Maurice Lagrandeur ${ }^{20}$, où Gahisto reconnaît des éléments de ce qui deviendra Le Cour serré). Pour Batouala, Maran précise qu'il se propose "d'attendre une nouvelle chasse au feu en décembre " pour revoir notamment l'avant-dernier chapitre "où il reçoit le coup de sagaie dont il mourra " (épisode dont nous savons qu'il fut modifié). Et Maran de se plaindre à nouveau : «ce roman-pénélope. Depuis 1911, j’y travaille. Depuis 1913-14 il est fini, archi-fini. Et toujours je trouve quelque chose à revoir... Quel cauchemar !» (p. 147).

Dans une lettre du 7 septembre 1918, Maran confie à Gahisto qu'il n'a pas le courage de chercher un éditeur - d'ailleurs il est « moins pressé pour l'argent » car ses frères ont trouvé des emplois. Dans une autre, trois semaines plus tard, il tient des propos contrastés sur l'opportunité d'une publication - vu le contexte de guerre - même si après avoir relu Batouala le mokondji (et non pas le «makondji », dit-il, comme il avait 
souvent orthographié le mot) «pour la première fois en entier depuis deux ans », il s'en déclare satisfait : «Je ne le croyais pas d'une si belle tenue. Je suis heureux d'avoir fait une œuvre » (p. 150). Mais ce n'est qu'après la joie de la nouvelle de l'armistice, en novembre, et d'autres empêchements, qu'il enverra les derniers chapitres du roman à Gahisto, début 1919.

Les dernières pages de l'article de Gahisto sont consacrées au retour de Maran en France en octobre 1919 et aux dernières corrections apportées au roman encore fin décembre, avant que Maran n'arrive à Paris, toujours indécis quant au sort de son manuscrit, début 1920. Gahisto cite alors la lettre du $1^{\mathrm{er}}$ mars, dans laquelle Maran lui raconte comment il s'est résigné à envoyer le manuscrit à Henri de Régnier ${ }^{21}$ après une galéjade avec son ami René Lafon, qui pourrait le faire passer pour un menteur. Alors que, dans cette lettre, Maran estime que, de toute façon, «Batouala, heureusement, ne sera pas pris ", Gahisto termine sa genèse du roman en mentionnant que le 17 mars, Maran lui annonçait «avoir reçu de très cordiales félicitations sur son manuscrit dont la publication était désormais assurée chez un grand éditeur parisien $»^{22}$. Et Gahisto ajoute que, si Maupassant avait déclaré avoir acquis ses notions de littérature en travaillant pendant sept ans avec Flaubert, René Maran avait lui achevé son livre en six ans, « seul dans la brousse, loin de toute tutelle de ce genre » (p. 154-155).

Pour conclure cette section, il convient : 1) de rappeler qu'il ne s'agit que d'un résumé de l'article de Gahisto et des citations des lettres qu'il contient (lesquelles ne sont qu'une petite sélection de l'ensemble de la correspondance Maran-Gahisto conservée dans le fonds d'archives familiales de l'écrivain); 2) de souligner que Gahisto ne mentionne pas la préface de Batouala dans sa genèse du roman. La genèse de cette préface sera au moins esquissée ci-dessous.

\section{Genèse de la préface de Batouala (1921)}

41 L'idée d'une préface pour le roman surgit tardivement : les documents consultables à ce jour la situent dans le congé de Maran en France entre octobre 1919 et début février 1921, plus précisément après la décision prise par Henri de Régnier de soutenir la publication du roman dont Maran venait de lui soumettre le manuscrit début mars 1920. Plus de six mois s'écoulent avant que Maran ne mentionne dans une lettre à Albin Michel, datée de Bordeaux le 18 octobre, que la rédaction d'une préface lui a été suggérée par Henri de Régnier et Jacques Boulenger. Un mois plus tard, le 14 novembre, Maran annonce à Albin Michel qu'il lui apportera la préface le lendemain : "J'ai vu Régnier ce matin ", précise-t-i ${ }^{23}$. On peut donc avancer que la préface a été rédigée entre mi-octobre et mi-novembre 1920, et qu'elle a été soumise à l'approbation de Régnier avant d'être remise en mains propres à l'éditeur.

Il n'y a pas lieu ici de procéder à une énième analyse de cette fameuse préface. Je souhaite plutôt esquisser les étapes du lent processus de maturation ${ }^{24}$ qui a conduit Maran à inclure dans ce texte les passages sulfureux qui allaient déchaîner les passions dans les institutions coloniales de la République et lui valoir d'être interpellé jusque devant l'Assemblée nationale. Mais avant de remonter aux premiers symptômes du malaise que l'administrateur colonial Maran allait développer parallèlement à sa découverte des sociétés indigènes de l'Oubangui-Chari, décrites dans le roman, je souhaite citer deux-trois lettres de l'écrivain, rédigées en hâte après sa révision des épreuves de Batouala et juste avant de s'embarquer pour son dernier voyage en Afrique. 

de Batouala. [...] Il va falloir que je m'occupe un peu de sa destinée avant mon départ. [...] Je vous prierai de bien vouloir me suppléer pour le service de presse... ». Le 21, nouvelle lettre: «J'aurais bien voulu pouvoir revenir à Paris avant de partir [vous revoir encore, vous et les vôtres, mais...]. / Avec Pujolle, je corrige Batouala. Ligne à ligne et mot à mot. [...] Chose extraordinaire, je trouve mon roman coloré, émouvant, rapide, substantiel, neuf. [...] La préface elle-même me plait. En supprimant quelques mots par ci par là, je lui ai donné une allure encore plus hostile. / Les coloniaux me voueront aux gémonies. Peu m'importe. Ce n'est pas pour les idiots que j'écris... » Puis une autre, le 26: «Lundi soir, sous pli recommandé, j'ai retourné à Albin Michel les premières épreuves de Batouala... [...]. D'ici mardi, je vous enverrai une [autre] lettre. J'y préciserai ce que j'attends de votre amitié pour Batouala... »

Cemarquable gestion par Maran des préparatifs de la publication de Batouala continue en effet dans la lettre dactylographiée de plusieurs pages du dimanche 30 janvier, deux jours avant de lever l'ancre. Il y précise notamment au point $4:$ «Je présente Batouala au prix Goncourt. Madame Gahisto voudra-t-elle être assez aimable pour me faire recommander par M. Dété aux trois "greniéristes" amis de ce dernier?", et au point 6: «Enfin, il vous faudra faire, au secrétariat de l'Académie française le dépôt de cinq exemplaires de Batouala [...] en mentionnant dans votre lettre - en mon nom - que vous désirez participer, avec cet ouvrage, au Grand Prix du Roman pour 1922. Voilà. Merci. Et... à dans quatre ou cinq ans. / René Maran ».

Si la boutade finale montre que Maran avait encore le sens de l'humour, fin janvier 1921, ces lettres montrent surtout que le romancier en herbe qui avait si longtemps hésité avant de proposer Batouala à un éditeur, avait suffisamment développé de confiance en lui-même depuis sa rencontre avec Régnier à Paris - ville qu'il affirmait à l'envi ne pas aimer - pour mettre son premier roman en lice dans les deux concours littéraires les plus prestigieux de la capitale, tout en ayant conscience que la préface ${ }^{25}$ risquait fort d'irriter - notamment dans les cercles dont dépendait sa carrière dans l'administration coloniale ${ }^{26}$.

La préface à Batouala, par l'auteur même, sert au moins quatre objectifs : 1) présenter une région particulièrement lointaine de l'Afrique et donc très peu connue; 2) expliquer les aspects littéraires d'un roman complètement original dans le champ de la littérature coloniale française de l'époque ; 3) dénoncer au moins les abus intolérables d'un système qui se targue hypocritement d'une mission civilisatrice louable pour couvrir une exploitation inhumaine; 4) régler des comptes personnels avec une machine administrative dont Maran a écrit plusieurs fois qu'elle l'avait «avili », lui, l'employé français mais noir de peau. Maran y interpelle d'emblée Henri de Régnier et Jacques Boulenger, "tuteurs de ce livre" (dédié à son "très cher ami, Manoel Gahisto »), avant d'en appeler au soutien de "mes frères en esprit, écrivains de France » pour qu'ils joignent leur voix à sa dénonciation d'infamies qui « déconsidèrent la nation dont [ils] sont les mainteneurs ».

Lorsque Maran rédige ses pages, il est en congé de convalescence en France et sait qu'il va retourner bientôt dans un service de l'administration coloniale où l'attend un lourd contentieux - qui va encore s'envenimer, suite au retentissement que l'attribution du Goncourt va accorder à la préface. Les passages dans lesquels il dénonce violemment les abus du système colonial et les aspects indignes de la vie dans les colonies d'Afrique reflètent, de manière très abrégée, les textes de nombreux rapports envoyés par Maran 
depuis plusieurs années à des supérieurs hiérarchiques divers mais aussi à des députés, et jusqu'au ministre des Colonies lui-même - souvent en réaction à des rapports faits sur son travail. Ces passages, les initiatives prises et les sentiments de Maran les concernant, sont aussi décrits dans d'innombrables lettres adressées à divers correspondants, si bien qu'on pourrait parler de deux types d'avant-textes de la préface. À défaut de pouvoir offrir ici une description génétique du processus, je vais citer, ou au moins mentionner, les références les plus importantes dans les correspondances de Maran, car les polémiques entre Maran et l'administration coloniale ont déjà fait l'objet d'une étude spécifique ${ }^{27}$.

Comme dans le dossier administratif de René Maran, les remarques critiques sur ses rapports avec la colonie apparaissent au plus tard en 1916 et vont se multipliant et s'intensifiant dans sa correspondance. Ainsi écrit-il le 11 octobre 1916 à Gahisto, que pour lui "l'Afrique Équatoriale est perdue ", livrée à la concussion et aux vols. En juin 1917, il confie au même Gahisto: "Je ne reviendrai plus à la colonie [...], on m'embête ", et le 27 décembre : «Je n'aime pas la colonie, parce que j'y ai trop souffert. [...] Je me révolte encore peut-être trop facilement ». Le 13 août 1917, il lui rapporte sur plusieurs pages un long entretien qu'il a eu avec le gouverneur Lamblin, à qui il avait déjà envoyé "une lettre au sujet de brimades administratives, dans laquelle je ridiculisais les méthodes tyranniques de ce Monsieur-là [un supérieur, comme lui d'origine antillaise, qui le déteste et lui fait subir depuis quatre ans un harcèlement au point « de me faire perdre mon sang-froid en mars dernier »]. Maran ajoute :

Nous nous séparâmes bons amis [...] mais je ne me fais pas d'illusion. Je n'aurai pas mon avancement en juillet. Qu'importe ! Les hautes sphères savent maintenant que lorsqu'on me marche sur les pieds, je crie [...]. On me laissera tranquille.

Dans une lettre de Bangui du 18 octobre 1918, Maran confie à Gahisto qu'il a « les nerfs sens dessus dessous. [...] Mon sort n'est pas bien fixé. Je suis à la fois proposé, d'une part pour des félicitations officielles, d'autre part pour un blâme officiel avec inscription au dossier ». Il reprend cette lettre le 2 novembre et, après avoir annoncé qu'il travaille au douzième chapitre de Batouala, ajoute :

On sait un peu partout à la colonie que j'ai quelque chose de bien dans mes papiers. On croit communément que c'est un pamphlet contre la vie administrative coloniale. Qu'ils attendent, ces idiots. Leur tour viendra. [...] Je n'ai pas encore dit ce que je pense. Je ne pense rien que d'exécrable. Les fonctionnaires m'ont appris à haïr. La haine, quelle belle passion !...

Ce genre de propos déprimés revient dans les lettres suivantes. Celle, très longue, du 16 novembre a valeur de testament :

Je peux disparaître d'un moment à l'autre. Je suis fatigué à l'extrême. La maladie a miné ma santé. La hantise de la mort me poursuit. J'ai donc pensé à vous faire don

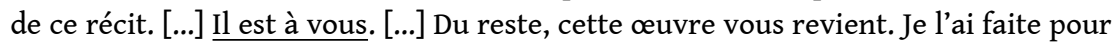
vous. [...] Car il vous faut croire en mon amitié. [...] Mon amertume d'autrefois, ma mélancolie sont devenues de l'hypocondrie. Et cela m'attriste.

Dans celle du 29 décembre, après avoir discuté d'aspects de Batouala et du Petit Roi de Chimérie, qu'il a déjà mis en chantier, il ajoute :

A côté de cela, je collige les pièces d'un volumineux réquisitoire que, par l'entremise de plusieurs députés coloniaux, je remettrai au Ministre des Colonies, peu après mon retour. Il y a des scandales qui doivent être au grand jour étalés. Je n'ai rien à perdre, mais tout à gagner. Ah! Ces fonctionnaires...

51 Ce passage formule clairement l'intention de Maran de dénoncer les abus de l'administration coloniale, dans l'esprit de ce qu'il allait résumer dans sa préface de 
Batouala. Dans la correspondance Maran-Gahisto à laquelle j'ai eu accès à ce jour, je n'ai pas trouvé de références plus précises aux initiatives prises par Maran auprès des députés des colonies Diagne et Candace, une fois qu'il était rentré en France. Il y en a peut-être dans ces échanges avec d'autres correspondants, puisque Maran résidait principalement à Bordeaux pendant ce long séjour.

Des mentions apparaissent aussi dans des lettres à son jeune ami Charles Barailley (que Maran tutoie, alors qu'il en restera toujours au vouvoiement avec Gahisto). Ainsi lui écrit-il le 10 février 1920, avec de légères touches flatteuses : « Hier, j’ai expédié à Henri de Régnier, qui a bien voulu me le demander, mon Batouala le Moukondji [...]. Enfin, il ne faut pas oublier la campagne coloniale que j'ai entreprise. M. Candace, député de la Guadeloupe, est de retour. Je vais, sans nul doute, lui servir de secrétaire bénévole. Ma vie se complique de plus en plus. Et c'est tant mieux...» (BBX2019-143/72232). Le 23 mars, il annonce à son ami que "Batouala doit paraître en feuilleton dans L'Opinion " et mentionne que Régnier a suggéré "quelques atténuations et suppressions dans le texte (1 page sur 156) » et de " voiler quelques crudités ».

Dans une autre lettre depuis Paris, non datée, il se plaint de ne pas avoir écrit une ligne depuis trois mois pour ses œuvres littéraires en cours, mais ajoute : « Entre temps, il est vrai, j'ai été pris par le terrible réquisitoire que j'ai dressé contre l'Oubangui-Chari et qui est entre les mains du Ministre des Colonies... » (BBX-72241). De retour en Afrique, il écrit à Barailley sur le bateau entre Brazzaville et Bangui le 3 mars 1921: " J'avais pris mes dispositions pour revenir dans l'Oubangui [mais je vais au Tchad]. Une colonie de choix. On n'y envoie que les pistonnés... » (BBX-148/72227).

Des informations similaires sont envoyées par Maran à Gahisto le 21 juillet 1921 (alors même que Batouala paraissait à Paris), depuis Fort-Archambault, où il a eu la satisfaction d'apprendre que "l'un des administrateurs contre lequel j'avais formulé des accusations implacables et précises, vient d'être, pour exaction, expulsé de l'administration. [...] d'autres ont été déplacés. Et l'on m'a envoyé au Tchad pour m'éloigner de la haine agissante de mes ennemis. [...] On me colle d'office la médaille coloniale que j'avais refusée [...] On m'accable de félicitations... ». Nous savons que ces satisfactions, dues certainement à l'intervention des députés coloniaux auprès du ministère suite au "réquisitoire » envoyé par Maran ${ }^{28}$, furent de courte durée, car la caisse de résonance du Goncourt allait bientôt mettre la préface incendiaire de Batouala sur la place publique et provoquer un terrible retour de bâton.

En guise de conclusion à cette étude, je voudrais simplement souligner que, sans l'écoute constante - critique et amicale - de Manoel Gahisto, depuis l'arrivée de René Maran en Afrique fin 1909 jusqu'à la sortie de Batouala en juillet 1921, il est probable que ce premier véritable roman nègre en langue française ne serait jamais paru. Et sa préface non plus. 


\section{BIBLIOGRAPHIE}

Fonds d'archives familiales René Maran.

Archives des bibliothèques municipales de Bordeaux

Dossier administratif de René Maran conservé aux ANOM.

Fonds « Hommage de Madame Camille René Maran à la République du Sénégal », conservé à la Bibliothèque centrale de l'UCAD de Dakar.

BOCQUET Léon, « Préface », dans René Maran, Le Petit roi de Chimérie, Paris, Albin Michel, 1924,

p. 7-63.

GAHISTO Manoel, « La genèse de Batouala », dans Hommage à René Maran, Paris, Présence Africaine, 1965, p. 93-155.

- - , « La genèse de Batouala », manuscrit UCAD, http://bibnum.ucad.sn/viewer.php?

$c=$ rares\& $d=1 \% 5 \mathrm{fms} \% 5 \mathrm{f} 24$.

MARAN René, Batouala, véritable roman nègre, Paris, Albin Michel, 1921.

- - Batouala, manuscrit, Archives des bibliothèques municipales de Bordeaux (BBX19104).

- - , Lettres à Charles Barailley, Archives des bibliothèques municipales de Bordeaux

- - Batouala, tapuscrit, Fonds d'archives familiales René Maran

- - Correspondance avec Manoel Gahisto, Fonds d'archives familiales René Maran

- - René Maran. Nouvelles africaines et françaises, inédites ou inconnues, édité par Roger Little,

L'Harmattan, coll. « Autrement mêmes », 2018.

RUBIALES Lourdes, « Désillusion et frustration : l'administration coloniale contre René Maran », (2009). http://www.sielec.net/pages_site/FIGURES/rubiales_maran.htm.

\section{NOTES}

1. René MARAN, La Maison du bonheur, Paris, Éditions du Beffroi. Quatre autres recueils suivront (en $1912,1922,1935$ et 1957).

2. Notamment dans le fonds André Fraisse (1909-1926), que Xavier Luce et Mbaye Gueye ont pu clicher pour l'ITEM.

3. Bien des aspects concernant ces genèses ont déjà été mentionnés dans d'importantes études consacrées à l'œuvre de René Maran. Elles sont nombreuses et je prie leurs auteur.e.s de m'excuser de ne pouvoir y référer par manque de place dans cet article. De même, je demande l'indulgence des lecteurs ou lectrices qui connaissent déjà certaines des sources recyclées ici.

4. Le tapuscrit de l'étude de Gahisto, conservé à l'UCAD de Dakar (ms_024), est cependant accessible en ligne; http://bibnum.ucad.sn/viewer.php?c=rares\&d=l\%5fms\%5f24. Précisons aussi que, dans la préface de Bocquet, seules les quatre dernières pages sur cinquante-six introduisent Le Petit Roi de Chimérie. Les cinquante-deux précédentes sont consacrées à une magnifique défense publique de l'écrivain René Maran, qui en avait bien besoin à cette époque.

5. Lesquelles comprennent notamment un gros dossier intitulé «Correspondance René Maran Manoel Gahisto » (projet d'édition en cours par le professeur Romuald Fonkoua), dans lequel de nombreuses lettres, non citées par Gahisto lui-même dans son article sur «La Genèse de 
Batouala", apportent encore bien des informations inédites sur le contexte de rédaction des œuvres de René Maran conçues pendant son séjour en AEF.

6. Il s'agit d'un projet d'édition critique à orientation génétique dans la collection «Planète Libre » de CNRS Éditions, mené par le groupe René Maran de l'équipe Manuscrits francophones de l'ITEM.

7. Sauf bévue, René Maran ne semble pas avoir relaté de souvenirs de sa première découverte de l'Afrique noire en tant qu'enfant, lorsque son père fut muté de Martinique au Gabon fin 1891. Il fallut d'ailleurs renvoyer en France le garçon, qui supportait mal le climat équatorial, et le mettre en pension à Bordeaux dès 1894.

8. Comme l'a formulé Claire Riffard après la présentation des notes pour cette étude lors du quatrième séminaire en ligne « Manuscrits francophones/René Maran » de l'ITEM le 9 avril 2021.

9. Il faut noter d'emblée que l'orthographe de ce terme africain varie dans les écrits de Maran comme dans ceux de Bocquet ou de Gahisto, notamment. On trouve aussi «Mokondji» ou «Mokoundji » - avec ou sans majuscule. Albin Michel retiendra «le mokoundji » dans l'édition de 1921 comme dans celle de 1938, dès la première page du roman. L'édition génétique tentera d'éclaircir cette question confuse.

10. Ce sera un point très important dans l'affaire Diagne-Maran, et dans «l'affaire Maran » de 1918, où Maran s'est vu accusé par des employés de l'administration coloniale d'avoir provoqué la mort de Mongo, un indigène, en le battant. Or, selon Maran, l'accusation résulterait d'une mauvaise traduction qu'un kapita aurait fait à un chef de poste. Il me paraît donc évident que la maîtrise du " petit nègre » était une forme de pouvoir, car les kapitas faisaient souvent office de traducteurs pour des chefs de poste blancs, ignorants des langues locales. Dans ses romans et contes, Maran a recours à un «très bon français » quand il narre les dialogues des animaux ou des indigènes s'exprimant en banda, langue difficile, que Maran a essayé de maîtriser, et dont il se fait le traducteur.

11. Bocquet cite une lettre de juillet 1914, dans laquelle Maran écrivait : « Je travaille la langue banda, bien plus difficile que le sango, cet espéranto de la rivière. J'ai colligé plus de trois cents mots, à peine le quart du vocabulaire que je voudrais rédiger » (p. 44). Ces cahiers de vocabulaire ont servi pour l'essai Légendes et coutumes nègres de l'Oubangui-Chari, choses vues (1933) et leurs manuscrits et tapuscrits sont accessibles dans la bibliothèque numérique de l'UCAD sous la cote Ms_32 (28 p. de ms + 22 p. de tps) et Ms_026 (en fait un tapuscrit des épreuves du livre, 86 p.).

12. Bocquet cite également une lettre d'octobre 1918 (année de la «mission Diagne» pour recruter des tirailleurs en Afrique), dans laquelle Maran exprimerait «sa vraie pensée » sur la question des tirailleurs noirs (et leur rôle dans la bataille de l'Yser), question qui, selon Maran, « dépasse de beaucoup la question des races [...] c'est celle du principe des nationalités dont on parle tant » (p. 48).

13. Cette aigreur est largement corroborée par les nombreuses pièces du dossier administratif de Maran, conservé aux ANOM. Selon certains supérieurs, Maran refusait de travailler et faisait « le poète décadent ». Il se plaignait de son côté d'un racisme primaire de la part de certains blancs et trouvait les tâches qu'on lui confiait inutiles. Mais il y a bien un agacement des deux côtés, qui allait s'amplifier après le «scandale de Batouala prix Goncourt ", et aboutir à " une faute grave » de Maran, puisqu'il quittera son poste au Tchad en 1923 sans autorisation hiérarchique.

14. Manoel Gahisto demeure un personnage un peu mystérieux. Ce nom d'écrivain est le pseudonyme de Paul Coolen (1878-1948), biographe, traducteur et critique littéraire, spécialisé en littératures lusophones. Sur la suggestion de Bocquet, Maran lui avait fait parvenir un exemplaire de son recueil de poèmes qui venait de paraître en 1909 , et leur correspondance de toute une vie allait commencer avec le mot de remerciement de Gahisto pour l'envoi du recueil et la réponse sous forme d'une carte postale envoyée par Maran, en route pour l'Afrique, depuis l'escale de Conakry. Assez rapidement, Gahisto, que ces lettres d'un continent qu'il ignorait ont fasciné, devient un interlocuteur apprécié et même un confident. La date de composition de 
l'étude sur «La genèse de Batouala » n'est pas mentionnée dans le scan du tapuscrit conservé à l'UCAD, mais elle semble avoir été suscitée par la sortie de la seconde édition de Batouala en 1938, qui lui reste dédiée. Un détail du texte (p. 96 de la version publiée) pointe à l'année 1940 pour sa mise en chantier. Il reste aussi une lettre de Gahisto essayant de la placer au Mercure de France en novembre 1947. Après sa mort, un an plus tard, c'est Maran qui rédigera une notice nécrologique sur son ami (coupure de presse sans indication de source dans les Archives familiales).

15. Le recueil paraîtra dès 1912 sous le titre La Vie intérieure. Le passage de Gahisto ne permet pas d'identifier clairement le destinataire de la lettre (Culine?) ou sa date de rédaction, mais c'est certainement en 1910.

16. Maran confie aussi qu'il venait seulement de développer une relation aimante avec «son pauvre papa ", pendant les six mois qu'ils passèrent à travailler ensemble à Grimari, et ajoute qu'au lieu de rentrer en France pour se marier, il travaillera pour faire vivre sa mère et ses frères (p. 101).

17. La pièce de théâtre ne sera jamais publiée mais le recueil Les Belles Images paraîtra en 1922.

18. Si bien que, du vivant de l'auteur, ce texte ne sera publié que sous forme réduite à une nouvelle dans Candide, journal hebdomadaire, no 199 ( 5 janvier 1928, p. 5 et 8). La version longue le sera dans Hommage à René Maran de 1965, op. cit., puis à nouveau dans le recueil René Maran. Nouvelles africaines et françaises, inédites ou inconnues, édité par Roger Little dans la collection « Autrement mêmes » chez L'Harmattan, 2018.

19. Ce que Gahisto ne mentionne pas dans ces pages - mais qui a été découvert récemment (en juillet 2021) par le Groupe René Maran grâce à Roger Little qui a eu l'amabilité de fournir des extraits plus substantiels et encore inédits de la correspondance Maran-Gahisto - c'est la dimension plus intime que prendra leur relation après des visites de Maran chez les Gahisto, rue Séguier, Paris $6^{\mathrm{e}}$, où il fait connaissance de Mme Gahisto et de sa jeune sœur, Bernerette, qui tombe amoureuse de Maran et avec qui Maran envisage de se fiancer - jusqu'en 1919. Dès son retour à Bordeaux fin septembre, des correspondances (avec envoi parfois de tapuscrits de chapitres) sont initiées entre Maran et les deux sœurs, parallèlement à celle maintenue avec Manoel Gahisto. Maran échangera dorénavant aussi avec ces femmes sur l'avancement de ses textes littéraires et leur publication.

20. La dimension autobiographique de ce nouveau projet de roman est d'autant plus évidente que le nom de jeune fille de la mère de René Maran était Lagrandeur. Je rappelle aussi que Maran avait évoqué un roman sur son enfance dès 1911. Ici, il lui donne un titre qu'il abandonnera plus tard.

21. Sauf bévue, l'origine de l'idée de contacter le poète académicien Régnier plutôt que de s'adresser à un éditeur reste obscure. Dans la même lettre, Maran mentionne aussi avoir, « ces quelques derniers jours, fait des démarches auprès de Jacques Rivière ", sans préciser leur résultat. Dans ce passage de son article, Gahisto passe très rapidement sur l'année 1919. Il n'y cite pas une lettre de lui-même à Maran du 23 août, qui montre qu'il s'était chargé (ou avait été...) de contacter des éditeurs potentiels, y compris des revues : «Jusqu'ici je n'ai pas remis Batouala au Mercure [...la revue ne prend plus de romans pour cette année »]. Gahisto ajoute d'ailleurs une question intéressante: "Comment aviez-vous pensé vous mettre en scène? C'est que cela ajouterait de l'intérêt à l'ouvrage. J'y songe avant de jouer la carte ». Comme Maran est totalement absent dans le roman, Gahisto songe manifestement à quelque chose comme une préface qui présenterait l'auteur, inconnu des lecteurs de romans français.

22. Maran a manifesté sa joie des félicitations reçues de Henri de Régnier et de Jacques Boulenger dans une lettre envoyée le jour même à Gahisto le 17 mars 1920. Il y précise aussi que : «C'est entendu. Je ferai les quelques légères corrections nécessaires ». Il n'est pas sûr que l'étude génétique du roman, en cours, puisse établir quelles corrections du manuscrit ont été suggérées par Régnier.

23. Archives Albin Michel, IMEC. 
24. Cette esquisse repose notamment sur l'accès à de nombreuses lettres encore inédites de la correspondance Maran-Gahisto, et ne pourra d'ailleurs être complétée que lorsque l'ensemble de ce fonds volumineux sera mis à la disposition des chercheur.e.s. Au nom du GRM je remercie vivement Roger Little d'avoir mis à disposition une sélection des passages concernant en particulier la genèse de Batouala.

25. Il est intéressant aussi de noter que la préface n'est pas mentionnée dans le «prière d'insérer " que Gahisto a envoyé à Albin Michel, sur la demande de ce dernier, juste avant la sortie du roman en juillet 1921. Par ailleurs, il reste à confirmer si ce document a effectivement été imprimé et inséré dans la première édition (archives privées).

26. Par la suite, Maran affirmera, dans une lettre à un certain Mualdes, qu'il voulait sciemment provoquer le ministère des Colonies avec sa préface: «Je me cache si peu de ce que l'on me reproche, que c'est pour déclencher une enquête [sur des accusations portées à son encontre qu'il estime injustes] que j'ai écrit la préface de Batouala». Voir note 39 dans "Désillusion et frustration : l'administration coloniale contre René Maran », par Lourdes RUBIALES (2009). http:// www.sielec.net/pages_site/FIGURES/rubiales_maran.htm.

27. Lourdes RUBIALES, art. cit.

28. Et dont on sait que le ministère allait prétendre qu'il n'avait jamais été reçu.

\section{RÉSUMÉS}

Il s'agit dans cette contribution de faire le point sur la genèse du roman Batouala de René Maran et la genèse de sa préface, rédigée par l'auteur lui-même, sur la base des documents disponibles dans diverses archives pour la période entre fin 1909 (départ de Maran pour l'Oubangui-Chari) et juillet 1921 (publication du roman par Albin Michel à Paris). Seront rappelés les passages les plus significatifs des études remarquables publiées sur ce thème par Léon Bocquet en 1924 et Manoel Gahisto en 1965, puis citées des correspondances encore inédites, notamment en ce qui concerne : 1) les initiatives prises par Maran et/ou Gahisto pour trouver un éditeur parisien ; 2) la rédaction de la célèbre préface; et 3) la présentation du roman pour l'attribution de prix littéraires - sur instructions de l'auteur, avant son retour précipité en Afrique en février 1921.

The point in this contribution is to update what is known about the genesis of René Maran's novel Batouala and the genesis of its preface, penned by the author himself. On the basis of documents available in various archives, between late 1909 (when young Maran left Bordeaux for the Oubangui-Chari) and July 1921 (when the novel was published by Albin Michel in Paris). The most significant passages of the remarquable studies published on this issue by Léon Bocquet in 1924 and Manoel Gahisto in 1965 will be brought back to memory and followed by a section quoting from hitherto unpublished correspondances with relevance to : 1) initiatives taken by either Maran or Gahisto to find a publisher for the novel in Paris ; 2) the writing of the famous preface; and 3) the entering of the novel in the competition for literary awards - upon instructions by the author, prior to his precipitated return to Africa in February 1921. 
INDEX

Mots-clés : Batouala, genèse, Gahisto Manoel, Bocquet Léon, Albin Michel, préface

Keywords : Batouala, genesis, Gahisto Manoel, Bocquet Léon, Albin Michel, preface

\section{AUTEUR}

\section{CHARLES W. SCHEEL}

Professeur émérite à l'Université des Antilles - Pôle Martinique. 